\title{
Myasthenia Gravis as a Cause of Failed Extubation Diagnosed by Diaphragmatic Ultrasound
}

\author{
Priya Ramakrishnan and Shahla Siddiqui
}

\begin{abstract}
Myasthenia Gravis (MG) is an autoimmune disease of the neuromuscular junction which results in generalised weakness. Presentation varies and can often be delayed as the course is waxing and waning. We report the case of an elderly patient who was admitted with severe cholangitis. The patient required a general anaesthesia with intubation for a planned endoscopic retrograde cholangiopancreatogram (ERCP). She was extubated a few hours after the procedure in the intensive care unit (ICU). However, a few hours later, she required re-intubation for tiring out and a raised $\mathrm{paCO}_{2}$ level. When assessed by a diaphragmatic ultrasound, she was found to have severe weakness, and a provisional diagnosis of MG was made after other causes, such as sepsis, were ruled out. As a fast, inexpensive and non-invasive test, ultrasound offers many advantages and can also be used serially to assess recovery of a paralyzed diaphragm as well to achieve successful liberation from mechanical ventilation.
\end{abstract}

Key Words: Myasthenia gravis, Diaphragm, Extubation.

\section{INTRODUCTION}

The diaphragm is the main muscle of respiration, and diaphragmatic excursion predicts successful liberation from mechanical ventilation. ${ }^{1}$ Myasthenia Gravis (MG) is a rare autoimmune disease of the neuromuscular junction which results in generalised weakness. ${ }^{2}$ Elderly patients with this disease are at particular risk from prolonged ventilation and extubation failure, which is common, with a high re-intubation rate. 3,4 Such patients need careful assessment and monitoring before liberation from mechanical ventilation. Traditionally, evaluation of diaphragmatic function is carried out by invasive and uncomfortable diaphragmatic electromyogram (EMG) tests. Alternatively, diaphragmatic ultrasound has been used to see excursion of the diaphragm and end expiratory thickness as a predictor of extubation. ${ }^{5}$ Using this modality, we can lower rates of unsuccessful extubation, cost effectively and quickly in the ICU. ${ }^{6}$ Studies have shown that in the static position, a diaphragmatic cut-off of diaphragm excursion for predicting weaning failure is $1.4 \mathrm{~cm}$ for the right hemidiaphragm, and $<1.4 \mathrm{~cm}$ excursion is consistent with a greater chance of weaning failure. ${ }^{7}$

\section{CASE REPORT}

We describe a case of an 89-year female who was admitted to the surgical ICU for cholangitis. She was previously well and fairly independent, given her age, with a past medical history significant for a cholecys-

Department of Anaesthesia, Khoo Teck Puat Hospital, Singapore

Correspondence: Dr. Shahla Siddiqui, Department of Anaesthesia,

Khoo Teck Puat Hospital, Singapore

E-mail:shahlasi@yahoo.com

Received: June 07, 2018; Accepted: October 20, 2018 tectomy and a humerus fracture. She gave no history of past neurological symptoms. Her abdominal pain and fever were only of 48 hours duration. After a successful endoscopic retrograde cholangiopancreato-gram (ERCP) procedure to remove her common bile duct stone and pus, she was shifted to the surgical ICU, intubated and mechanically ventilated. Three hours later, when she was awake and met the criteria for extubation, her endotracheal tube was removed successfully. At this point, her breathing rate, heart rate and temperature were normal. However, soon after extubation, her arterial blood gas $\mathrm{CO}_{2}$ rose and she became drowsy, requiring reintubation. All other causes of failed extubation, such as sepsis and pneumonia, were ruled out. After a few days, when her serum inflammatory markers settled, it was noted that her respiratory excursion was still low. A diaphragmatic ultrasound on spontaneous ventilation showed no movement of the diaphragm (minimal diaphragmatic excursions) and reduced thickness at end expiration $(<1 \mathrm{~cm}$, Figures 1 and 2). She had not been sedated or paralysed recently. The family further gave a previous history of ptosis. A neurology opinion was sought, and after an EMG test proved positive for severe myopathy, a diagnosis of MG was made. Serum markers were subsequently positive as well. Treatment was started with pyridostigmine as well as intravenous immunoglobulins (IVIG). She underwent a tracheostomy subsequently, due to prolonged ventilation and slowly weaned.

\section{DISCUSSION}

The diaphragm is the major muscle used for respiration. Dysfunction can be caused by systemic conditions that cause muscle weakness. However, evaluation of diaphragmatic function by means of EMG, is difficult to 


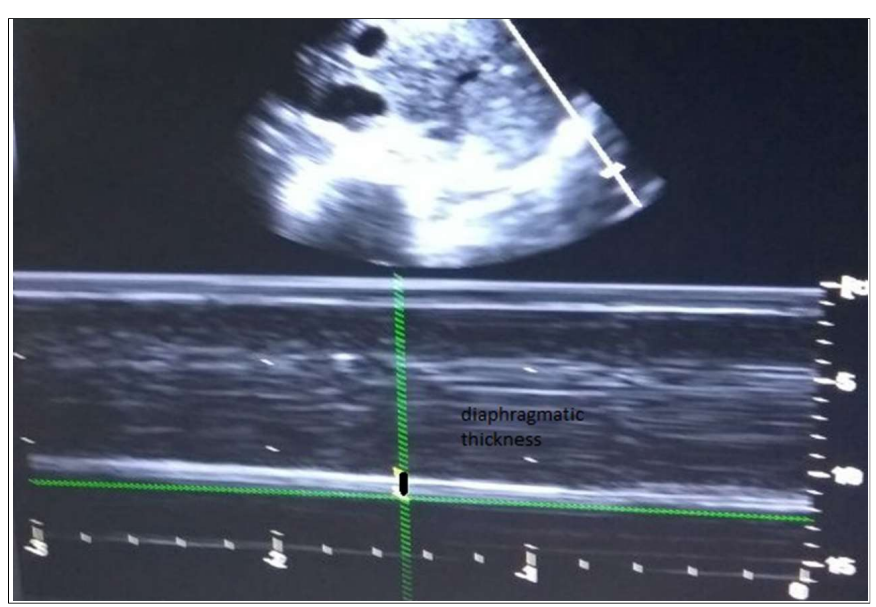

Figure 1: Ultrasound of diaphragm thickness.

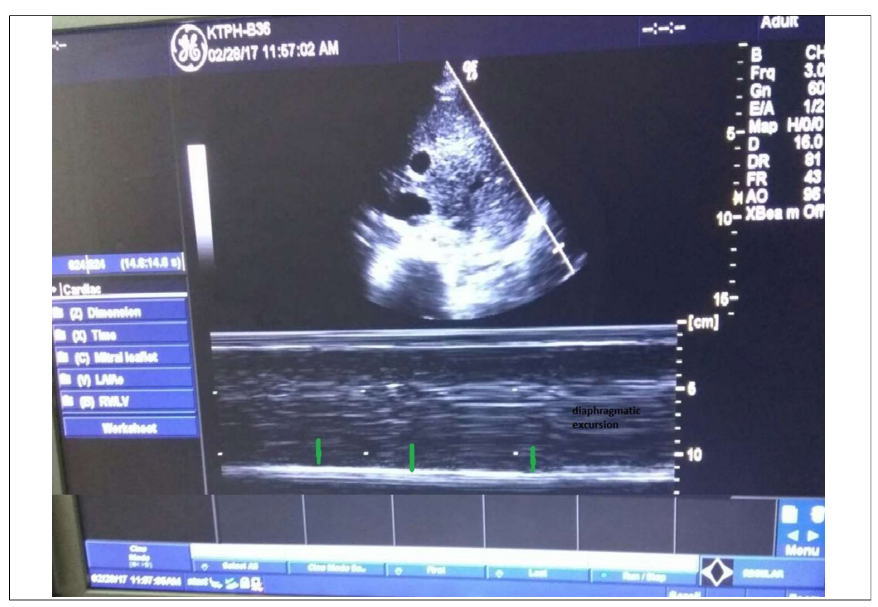

Figure 2: Ultrasound of diaphragm excursion.

do and costly. Neuromuscular ultrasound of the diaphragm is an evolving diagnostic modality with several techniques and measurements that can be employed for structural and functional assessment of the diaphragm. A novel use of this technique can be the prediction of successful extubation, with good sensitivity and specificity, and may also be a useful adjunct to diagnosing diaphragmatic paralysis from neuromuscular diseases, such as MG.

The diaphragm is the primary muscle of respiration and can be atrophied by offloading during mechanical ventilation. Prolonged ICU mechanical ventilation in patients with myopathies and neuropathies lead to added complications and cost. Using the ultrasound to assess diaphragmatic function at point of care can be a readily available tool to predict successful extubation and strength of the muscle during weaning. EMG and other tests may be time-consuming and costly, which increases the efficacy of a non-invasive and easily available test such as a diaphragmatic ultrasound. Figure 1 shows an image in the $\mathrm{M}$ Mode using a curvilinear probe, the right diaphragmatic thickness (less than $1 \mathrm{~cm}$ ), and Figure 2 shows a poor diaphragmatic excursion (less than $1 \mathrm{~cm}$ ), which predicts a high probability of extubation failure.

As a fast, inexpensive and non-invasive test, ultrasound offers many advantages and can also be used serially to assess recovery of a paralysed diaphragm as well to achieve successful liberation from mechanical ventilation.

\section{REFERENCES}

1. Boon AJ, Harper CJ, Ghahfarokhi LS, Strommen JA, Watson JC, Sorenson EJ. Two-dimensional ultrasound imaging of the diaphragm: Quantitative values in normal subjects. Muscle Nerve 2013; 47:884-9.

2. Boussuges $A$, Gole $Y$, Blanc P. Diaphragmatic motion studied by m-mode ultrasonography: methods, reproducibility, and normal values. Chest 2009; 135:391-400.

3. Hellyer NJ, Andreas NM, Bernstetter AS, Cieslak KR, Donahue GF, Steiner EA. Comparison of diaphragm thickness measurements among postures via ultrasound imaging. PM R 2017; 9:21-5.

4. Summerhill EM, El-Sameed YA, Glidden TJ, McCool FD. Monitoring recovery from diaphragm paralysis with ultrasound. Chest 2008; 133:737-43.

5. Ferrari G, De Filippi G, Elia F, Panero F, Volpicelli G, Aprà F. Diaphragm ultrasound as a new index of discontinuation from mechanical ventilation. Crit Ultrasound J 2014; 6:8

6. Kim WY, Suh HJ, Hong SB, Koh Y, Lim CM. Diaphragm dysfunction assessed by ultrasonography: Influence on weaning from mechanical ventilation. Crit Care Med 2011; 39:2627-30

7. Sarwal A, Walker FO, Cartwright MS. Neuromuscular ultrasound for evaluation of the diaphragm. Muscle Nerve 2013; 47:319-29. 